\title{
MEASURABLE HOMOMORPHISMS OF LOCALLY COMPACT GROUPS
}

\author{
ADAM KLEPPNER
}

(Communicated by Jonathan M. Rosenberg)

\begin{abstract}
Let $G$ and $H$ be locally compact groups and $\varphi$ a homomorphism from $G$ into $H$. Suppose that $\varphi^{-1}(U)$ is measurable for every open set $U \subset H$. It is known under some conditions, for example, if $H$ is $\sigma$-compact, that $\varphi$ is continuous. Here it is shown that this result is true without any countability restrictions on $G$ and $H$. The proof depends on the observation that the regular representation of $H$ is a homomorphism.
\end{abstract}

There are a variety of results which assert that a homomorphism between two topological groups which is measurable in one sense or another is continuous. Perhaps the oldest of these is a result of Banach [1, Chapter 1, Theorèm 4] which states that a Borel measurable homomorphism between polonaise groups is continuous. For locally compact groups it is known that a Haar measurable homomorphism into a $\sigma$-compact group is continuous [5, 22.18]. Recently, Moskowitz, making ingenious use of unitary representations, has given another proof of this [6, Theorem 1]. Here, we want to show that this result is true without any countability restrictions on the groups.

The measures referred to throughout are left Haar measures on the appropriate groups.

Theorem 1. Let $G$ and $H$ be locally compact groups and $\varphi: G \rightarrow H$ a homomorphism with the property that $\varphi^{-1}(U)$ is measurable for every open subset $U$ of $H$. Then $\varphi$ is continuous.

We can relax the requirement that $\varphi$ be a homomorphism, but not without requiring something additional.

Theorem 2. Let $G$ and $H$ be locally compact groups and $\varphi: G \rightarrow H$ a map which satisfies:

(1) $\varphi(x) \varphi(y)=\varphi(x y)$, locally almost all $x, y \in G \times G$, and $\varphi\left(x^{-1}\right)=$ $\varphi(x)^{-1}$, locally almost all $x \in G$.

Received by the editors June 16, 1987 and, in revised form, July 1, 1988.

1980 Mathematics Subject Classification (1985 Revision). Primary 22D05. 
(2) $\varphi^{-1}(U)$ is measurable for every open set $U \subset H$.

(3) There is an open relatively compact neighborhood $V$ of $e \in H$ so that $\varphi^{-1}(V)$ is not locally null.

Then there is a continuous homomorphism $\varphi_{*}: G \rightarrow H$ so that $\varphi(x)=\varphi_{*}(x)$, lae.

For brevity, we shall say that a function $f$ with the property that $f^{-1}(U)$ is measurable for all open $U$ is semimeasurable. In general, semimeasurable functions are not measurable [2, Chapter IV, §5].

We first prove Theorem 2 and begin the proof with the following observation, which is certainly not new.

Lemma 1. The left regular representation $\lambda$ of $H$ is a homeomorphism into the group $\mathscr{U}\left(L^{2}(H)\right)$ of unitary operators on $L^{2}(H)$ provided with the strong operator topology.

Proof. Since $\lambda$ is continuous and faithful, we must show that $\lambda$ is open, and because $\lambda$ is an isomorphism it is enough to show that $\lambda(V)$ is a neighborhood of 1 in $\lambda(H)$, for every neighborhood $V$ of $e$ in $H$. This is equivalent to showing that if $\left\{y_{\alpha}\right\}_{\alpha \in A}$ is a net in $H$ which does not converge to $e$, then $\left\{\lambda\left(y_{\alpha}\right)\right\}_{\alpha \in A}$ is a net which does not converge to 1 . But if $y_{\alpha} \nrightarrow e$, there is a neighborhood $V$ of $e$ in $H$ and a subnet $\left\{y_{\alpha(\beta)}\right\}_{\beta \in B}$ so that $y_{\alpha(\beta)} \notin V$, $\beta \in B$. Choose a neighborhood $W$ of $e$ in $H$ so that $W W^{-1} \subset V$. Let $f$ be the characteristic function of $W$. Then $\left(\lambda\left(y_{\alpha(\beta)}\right) f, f\right)=0$, all $\beta \in B$, and this implies $\lambda\left(y_{\alpha(\beta)}\right) \nrightarrow 1$.

Now suppose $\varphi$ satisfies the hypotheses of Theorem 2. Then $x \rightarrow \lambda(\varphi(x))=$ $\pi(x)$ is a map of $G$ into the group of unitary operators on $L^{2}(H)$ with the property that for all $u, v \in L^{2}(H), x \rightarrow(\pi(x) u, v)$ is measurable. For $f \in L^{1}(G)$ put $\pi(f)=\int_{G} \pi(x) f(x) d x$. The usual computations show that $f \rightarrow \pi(f)$ is a $*$ representation of $L^{1}(G)$. Let us show it is nondegenerate. Choose $u \in L^{2}(H), u \neq 0$. There is an open relatively compact neighborhood $U$ of $e \in H, U \subset V$ so that $h(y)=(\lambda(y) u, u) \neq 0, y \in U$. Choose an open neighborhood $U_{1}$ of $e$ in $H$ so that $U_{1} U_{1}^{-1} \subset U$. Since $V$ is relatively compact, there are $y_{1}, \ldots, y_{n} \in H$ so that $V \subset \bigcup_{1}^{n} U_{1} y_{k}$, and $\varphi^{-1}(V) \subset \bigcup_{1}^{n} \varphi^{-1}\left(U_{1} y_{k}\right)$. Since $\varphi^{-1}(V)$ has positive measure, for at least one $k, \varphi^{-1}\left(U_{1} y_{k}\right)$ has positive measure as does $\varphi^{-1}\left(U_{1} y_{k}\right) \varphi^{-1}\left(U_{1} y_{k}\right)^{-1}$. For locally almost all $x_{1}, x_{2} \in \varphi^{-1}\left(U_{1} y_{k}\right)$,

$$
\varphi\left(x_{1} x_{2}^{-1}\right)=\varphi\left(x_{1}\right) \varphi\left(x_{2}\right)^{-1} \in U_{1} y_{k}\left(U_{1} y_{k}\right)^{-1} \subset U .
$$

Thus $\varphi^{-1}(U)$ contains all of $\varphi^{-1}\left(U_{1} y_{k}\right) \varphi^{-1}\left(U_{1} y_{k}\right)^{-1}$, except for a locally null set, and has positive measure. Let $K$ be a compact subset of $\varphi^{-1}(U)$ of positive 
measure, and put

$$
f(x)= \begin{cases}(u, \lambda(\varphi(x)) u), & x \in K \\ 0, & x \notin K\end{cases}
$$

Then

$$
(\pi(f) u, u)=\int_{K}|(\lambda(\varphi(x)) u, u)|^{2} d x>0 .
$$

Thus $\pi(f) u \neq 0$. This shows $\pi$ is nondegenerate. It is well known $[3,13.3 .4]$ that there is a continuous unitary representation $\pi_{*}$ of $G$ so that $\pi(f)=$ $\int \pi_{*}(x) f(x) d x$, all $f \in L^{1}(G)$. It follows from this that $\pi(x)=\pi_{*}(x)$, lae. Put $G_{1}=\pi_{*}^{-1}(\lambda(H)) . G_{1}$ is a subgroup of $G$ and because $\pi=\pi_{*}$ lae, $G_{1}$ has a locally null complement. Such a subgroup is the whole group. We can now define $\varphi_{*}$ by putting $\varphi_{*}(x)=\lambda^{-1}\left(\pi_{*}(x)\right) \cdot \varphi_{*}$ is a continuous homomorphism which agrees with $\varphi$ lae.

To prove Theorem 1 we shall show that if $\varphi$ is a semimeasurable homomorphism, there is a relatively compact neighborhood $V$ of $e$ in $H$ so that $\varphi^{-1}(V)$ is not locally null. It then follows from Theorem 2 that there is a continuous homomorphism $\varphi_{*}$ so that $\varphi_{*}=\varphi$ lae. But now $\varphi$ and $\varphi_{*}$ agree on a subgroup with a locally null complement, and the only such subgroup is the whole group.

For the proof we need to know the existence of certain nonmeasurable sets. We use $\infty$ to denote a countable infinity.

Lemma 2. The following conditions on a subgroup $N$ of $G$ are equivalent:

(1) for all $x \in G,\left[N: x N x^{-1} \cap N\right] \leq \infty$,

(2) each double coset $N x N$ is a union of countably many left $N$ cosets,

(3) each double coset $N x N$ is a union of countably many right $N$ cosets,

(4) for each $x \in G$ there is a countable set $D$ so that $N x \subset D N$.

(5) for each $x \in G$ there is a countable set $D^{\prime}$ so that $x N \subset N D^{\prime}$,

(6) if $C$ is a countable subset of $G$ and $M$ is the subgroup generated by $N \cup C$, then $[M: N] \leq \infty$.

Proof. The double coset $N x N$ is the orbit under right translation by $N$ of $N x \in N \backslash G$. The stabilizer is $x N x^{-1} \cap N$. This shows (1) $\rightarrow$ (2). Clearly, $(2) \rightarrow(4)$ and $(3) \rightarrow(5)$. The map $x \rightarrow x^{-1}$ shows that $(4) \rightarrow(5)$. To show these imply (6) it is enough to find a subgroup $M_{1} \supset M$ so that $\left[M_{1}: N\right] \leq \infty$. Replacing $C$ by $C \cup C^{-1}$, we may suppose $C=C^{-1}$. Since $C=C^{-1}$, the subgroup $M_{1}$ generated by $N \cup C$ is the union of all sets of the form $N c_{1} N c_{2} \cdots c_{k} N$. By induction on $k$, using (3), it can be proved that each such set is a countable union of right $N$ cosets. Thus $M_{1}$ is a countable union of right $N$ cosets. Finally, suppose (6) holds. Choose $x \in G$ and let $M$ be the subgroup generated by $x$ and $N$. Since $M$ is a union of countably many $N$ cosets, so is $N x N$ which is contained in $M$. 
We shall say that a subgroup with any of these properties is asoo (a term suggested by $R$. Lipsman). Of course, countable or normal subgroups are asoo. An open $\sigma$-compact subgroup is asoo. If $p: G \rightarrow H$ is a homomorphism and $L$ is asoo in $H$ then $p^{-1}(L)$ is asoo in $G$. If $p$ is onto and $N$ is asoo in $G$, then $p(N)$ is asoo in $H$. If $N$ is asoo in $G$ and $C$ is a countable subset of $G$, the group generated by $N \cup C$ is asoo.

Lemma 3. Let $G$ be a compactly generated locally compact group and $N$ a null asoo subgroup of $G$. Then there is a nonmeasurable set $S \subset G$ so that $S=N S$.

Proof. In the following, $\mu$ denotes a left Haar measure. We begin with the case that $G$ is separable. (It is not necessary here to assume $G$ is compactly generated.) Let $U_{1} \supset U_{2} \supset \cdots$ be a basis for the neighborhoods of $e$. Since no finite union of $N$ cosets contains a nonempty open set, we may choose elements $x_{0}, x_{1}, \cdots$ as follows. Put $x_{0}=e$. Having chosen $x_{0}, x_{1}, \ldots, x_{n}$, we choose $x_{n+1} \in U_{n+1}-\bigcup_{1}^{n} x_{i} N$. Let $M$ be the group generated by $N \cup\left\{x_{1}, x_{2}, \ldots\right\}$, and let $Y$ be a set of right coset representatives of $M$ in $G$. Put $S=N Y$. Suppose $S$ is measurable. If $X$ is a set of left coset representatives of $N$ in $M$ then $X$ is countable and $G=X S$. Since $\mu(G) \leq \sum_{x \in X} \mu(x S) \leq \infty \mu(S)$, $\mu(S)>0$. Let $F \subset S$ be a set of finite positive measure. For some $n$, the function $x \rightarrow \mu(F \cap x F)>0$ for all $x \in U_{n}$. But $F \cap x_{n} F \subset S \cap x_{n} S=\varnothing$, and this is a contradiction. Thus $S$ is nonmeasurable.

We next consider the case that $N$ is not closed in $G$. Let $C$ be a countably infinite subset of $\bar{N}-N$, let $M$ be the subgroup generated by $N \cup C$, let $X$ be a set of left coset representatives of $N$ in $M, Y$ a set of right coset representatives of $M$ in $G$. Because $N$ is asoo, $X$ is countable. Put $S=N Y$. If $x \in X-N$, $x N S \cap S=\varnothing$. Suppose $S$ is measurable. Because $G=X S, S$ must have positive measure. Let $F \subset S$ be a set of finite positive measure. The function $x \rightarrow \mu(F \cap x F)$ is continuous and positive at $e$. Thus there is a neighborhood $U$ of $e$ so that $\mu(F \cap z F)>0$, all $z \in U$. If $x \in X-N$, the coset $x N$ is dense in $\bar{N}$, so that $x N \cap U \neq \varnothing$. Thus there is $y \in U \cap x N$ with $\mu(F \cap y F)>0$, but $y=x n$ for some $n \in N$, and $F \cap y F \subset S \cap x n S=\varnothing$, which is a contradiction.

To finish the proof, we need to consider the case that $N$ is closed. Because $G$ is compactly generated, there is a family $\left\{K_{\alpha}\right\}$ of compact normal subgroups of $G$ so that $G / K_{\alpha}$ is separable, and $G=\lim _{\alpha} G / K_{\alpha}$ [4, Theorem 3]. Let $p_{\alpha}$ be the canonical map of $G$ on $G / K_{\alpha} \cdot \overleftarrow{p_{\alpha}(N)}$ is asoo. If for some $\alpha$, $p_{\alpha}(N)$ is null, then by the first part of the proof there is a nonmeasurable set $S_{1} \subset G / K$ so that $S_{1}=p_{\alpha}(N) S . S=p_{\alpha}^{-1}\left(S_{1}\right)$ is the desired nonmeasurable set. What remains is the case that $N$ is closed and there is a compact normal subgroup $K$ such that $N K$ is open. Then $N K / N$ is infinite. Let $C$ be a countable subset of $N K$ whose image in $N K / N$ is infinite, and let $M$ be the subgroup of $N K$ generated by $N \cap C . M$ is asoo. If it is closed in $N K$, $M / N$ is closed in $N K / N$, and because $[M: N] \leq \infty, N$ is open in $M$. Thus $M / N$ is a discrete, closed subset of the compact space $N K / N$, thus finite. This contradicts the choice of $C$. Thus $M$ is not closed, and by the second part of 
the proof there is a nonmeasurable set $S \subset N K$ so that $S \subset N S \subset M S=S$. Since $N K$ is open in $G, S$ is nonmeasurable in $G$. This completes the proof.

With a little bit more work, one can show that if $N$ is a null subgroup of $G$ and there is a compactly generated open subgroup $G_{0} \subset G$ so that $N \cap G_{0}$ is asoo in $G_{0}$, for example, if $N$ is normal in $G$, then there is a nonmeasurable subset $S \subset G$ so that $N S=S$.

We can now complete the proof of Theorem 1. Let $\varphi: G \rightarrow H$ be a semimeasurable homomorphism. To show $\varphi$ is continuous it is enough to show that the restriction of $\varphi$ to any open subgroup is continuous. Thus we may replace $G$ by an open compactly generated subgroup and assume that $G$ is compactly generated. Suppose there is an open relatively compact neighborhood $V$ of $e$ in $H$ so that $\varphi^{-1}(V)$ is null. Let $L$ be an open $\sigma$-compact subgroup of $H$. Since $L$ is contained in the union of countably many translates of $V, N=\varphi^{-1}(L)$ is contained in the union of countably many translates of $\varphi^{-1}(V)$, and is null. Because $L$ is asoo in $H, N$ is asoo in $G$. By Lemma 3 , we know there is a nonmeasurable subset $S \subset G$ so that $N S=S$. Let $T=\varphi(S) . L T$ is open in $H$ and by hypothesis, $\varphi^{-1}(L T)$ is measurable. But $S \subset \varphi^{-1}(L T)=\varphi^{-1}(L) \varphi^{-1}(T) \subset N(N S)=S$. This is a contradiction. Thus $\varphi^{-1}(V)$ is not null. We now know from the remarks at the beginning of this section that $\varphi$ is continuous.

\section{ACKNOWLEDGMENTS}

I am indebted to Jack Feldman for suggesting a key idea in the proof of Lemma 3, to Esben Kehlet who noted a gap in my original proof of Lemma 3 and pointed out how to use nonclosed subgroups, and to the referee for suggesting a shorter proof of one of the implications in Lemma 2.

\section{REFERENCES}

1. S. Banach, Théorie des operations lineaires, Garasirski, Warsaw, 1932.

2. N. Bourbaki, Intégration, Hermann, Paris, 1956, 1963.

3. J. Dixmier, Les $C^{*}$ algébres et leurs représentations, 2nd. ed., Gauthier-Villars, Paris, 1969.

4. V. M. Glǔskov, Locally compact groups and Hilberts fifth problem, Amer. Math. Soc. Transl., Ser. 2, 15 (1960), 55-94.

5. E. Hewitt and K. Ross, Abstract harmonic analysis I. Springer-Verlag, New York, 1963.

6. M. Moskowitz, Uniform boundedness for non-abelian groups, Math. Proc. Cambridge Phil. Soc. 97 (1985), 107-110.

Department of Mathematics, University of Maryland, College Park, Maryland 20742 